\title{
Simulation study of relief goods delivery and information sharing by epidemic transmission in disaster areas
}

\author{
Kazuyuki Miyakita ${ }^{1}$, Keisuke Nakano ${ }^{2, *}$, Masashi Yamashita ${ }^{2}$, Hiroshi Tamura $^{3}$ \\ ${ }^{1}$ Center for Academic Information Service, Niigata University, Niigata, 950-2181 Japan \\ ${ }^{2}$ Department of Information Engineering, Niigata University, Niigata, 950-2181 Japan \\ ${ }^{3}$ Department of Electrical, Electronic and Communication Engineering, Chuo University, \\ Tokyo, 112-8551 Japan \\ *nakano@ie.niigata-u.ac.jp
}

Received: August 19, 2015; Accepted: January 18, 2016; Published: August 8, 2016

\begin{abstract}
During large-scale disasters, some regions can become isolated from critical information exchanges due to problems with communication infrastructures. In this paper, we consider information exchange among isolated shelters in such cases using epidemic transmission, which distributes information without infrastructure by direct communication between nodes and their movements. As mobile nodes for epidemic transmission, we consider transport vehicles that are leaving a distribution center to deliver relief goods to shelters. These transport vehicles receive information from the shelters and distribute it to other shelters together with the relief goods. As a result, information is shared by the shelter. Suppose that transport vehicles move along different traveling paths and exchange information at the distribution center. Transport vehicles can also get new information from other transport vehicles while they wait for new relief goods to arrive at the distribution center. Transport vehicles might be able to receive more new information by waiting for a longer time, although such additional waiting time causes a greater delay of the delivery of relief goods. In this paper, we consider the relation between relief goods delivery and information sharing by transport vehicles with epidemic transmission and analyze how the additional waiting time affects the delay of relief goods and the time needed for information sharing by computer simulation.
\end{abstract}

Keywords: Large-scale disasters, Inter-region communication, Goods transportation, Delay tolerant networks

\section{Introduction}

In emergent situations such as disasters, information systems play important roles in not only immediate response and long-term recovery but also awareness and prevention of such 
situations. Such information systems for emergent situations are called emergency management information systems (EMISs) and have been studied in various aspects as follows [四:

- Design (e.g., framework and user interface) of EMISs

- Ethical issues in EMISs

- Efficient use of EMISs

- Simulation of emergent situations

- Use of geographical information systems (GISs)

- Use of space technologies (e.g., satellite imagery)

- Standards-based resource emergency management systems

- Environmental risk management information systems

- Use of social computing systems

- Case studies

- Other important issues

It is an important role of EMISs including information and communication technologies to save and preserve human lives, infrastructure, and the environment [四].

Mobile communication systems are important infrastructure to be preserved in our society. A cellular mobile communication system consists of a backbone network and base stations. Such an infrastructure-based communication network might become disabled during large earthquakes because of the backbone network's failure due to power outages, the collapse of buildings, and so on. Hence, an alternative communication way during disasters must be provided, and many trials have been done [W]-[[]]. Multi-hop wireless networking is one effective kind of networking in disaster situations because mobile nodes construct a multi-hop wireless path between a source and a destination without any infrastructure. Multi-hop networking is sometimes used with additional equipment if the density of the nodes is insufficient to construct a connected multi-hop path, although such a situation with a low density of nodes is expected in disaster situations. For example, flying relay stations were introduced as additional equipment to improve connectivity performance [B]].

During disasters, situations sometimes arise where information delivery is more important than latency and long waiting times are acceptable. In such situations, we have another mobile communication method: epidemic transmission [6]. In epidemic transmission, unlike multi-hop networking, a source and a destination do not require a connected multi-hop path, and the source immediately sends information to the neighbor nodes. Mobile nodes that receive information repeatedly send it to other mobile nodes while moving. As a result, the information is spread and delivered to a destination. Epidemic transmission is an important technology to realize a delay tolerant network (DTN), which accepts a long delay for information exchanges in difficult situations [0],[[]]. 
In this paper, we use epidemic transmission to share information among isolated shelters in disaster situations. During disasters, since people are often required to stay at shelters, they might have difficulty traveling between such places. Even under such situations, however, vehicles transporting relief goods travel to shelters. Hence, we propose the use of vehicles that transport relief goods to shelters as special nodes to realize epidemic transmission in disaster situations. We consider information delivery to such shelters by the transport vehicles of relief goods using epidemic transmission. The transport vehicles for epidemic transmission play a similar role to the flying relay nodes in multi-hop networks in the above example from the viewpoint of performance enhancement.

We consider information exchanges among shelters that are isolated due to the breakdown of communication infrastructure. Suppose that a transport vehicle departs a distribution center to deliver relief goods to shelters. At the same time, the transport vehicle collects information from the shelters and delivers it by epidemic transmission with the relief goods. As a result, information is shared among the shelters. If some transport vehicles move along different traveling paths to deliver relief goods, these vehicles have to exchange information so that all of the shelters share information. For this, we assume that transport vehicles exchange information at the distribution center because they load new relief goods at the distribution center and can exchange information while loading. If a transport vehicle waits for new relief goods to arrive at the distribution center, it can meet other transport vehicles at the center and perhaps receive newer information from other transport vehicles. From another viewpoint, the transport vehicle might receive more new information by waiting for a longer time than the time needed for loading relief goods, although this additional waiting time causes more delay of the delivery of relief goods. In this paper, we analyze how the additional waiting time affects the delay of relief goods and the time needed for information sharing by computer simulation.

To do this, we consider the following three mean values for the performance metrics: (1) the mean time interval of the arrival of relief goods at a shelter; (2) the mean time interval of the arrival of information at a shelter; and (3) the mean delay of the arrival of information, which is the interval from the time when it is generated to when it arrives at a shelter. We compute the above three values by computer simulation and address their relation to the additional waiting time at the distribution center to increase information exchanges among transport vehicles. In this evaluation, we investigate an extreme behavior of performance by focusing on the properties of these mean values in the following two extreme cases:

- Case 1: The additional waiting time is 0.

- Case 2: The additional waiting time is sufficiently large.

Throughout this evaluation, we show some of the properties of the relation of the delay of the delivery of relief goods and the information sharing performance to additional waiting time.

Concerning related works, efficient scheduling methods for transporting relief goods in disaster situations have been widely studied [0]-[15]. Many trials provide an alternative communication method when communication infrastructure is disabled by disasters [四]-[[]]. However, few papers have studied the relation between the efficiency of the delivery of 
relief goods and the delivery of information based on a situation where relief goods and information are simultaneously delivered. We address a situation where transport vehicles deliver information about shelters together with relief goods and analyze the relation among the efficiencies of such deliveries.

In Section Q 2 , we explain our model that analyzes the relation between the additional waiting time and the delay of the delivery of relief goods and another relation between the additional waiting time and the information sharing performance during disasters where transport vehicles navigate on different traveling paths to visit shelters. In Section B, we explain some assumptions and parameters used for our computer simulation. In Section 团, we compute the three performance metrics by computer simulation and discuss how these values are affected by the additional waiting time at the distribution center. In Section [1, we conclude this paper.

\section{Delivery of relief goods and information by transport vehicles}

\subsection{Delivery of relief goods}

Assume $n$ shelters and a distribution center in the service area. Relief goods periodically arrive at the distribution center. Transport vehicles move along a road network that connects the shelters (Fig. W). In the case of disasters, some roads can be unavailable. In fact, the unavailable roads were found by probe car systems [ए]], which are navigation systems using mobile communication and positioning by GPS, in the Great East Japan Earthquake in 2011 [[7]. The founded unavailable roads were opened to the public as a map together with available roads. As can be seen from this fact, the road network can become disconnected due to the unavailable roads, and some shelters can be isolated. In this paper, however, we pay attention to a connected road network as the first step of this research. Considering epidemic transmission in disconnected networks with unavailable roads is a future problem. $m$ transport vehicles move along different paths. Assume $m$ different paths are designed in advance. A transport vehicle picks up relief goods at the distribution center and delivers them to the shelters on the traveling path assigned to this vehicle. $t_{\text {stay }}$ is the time needed for loading and unloading at one place. If there are no relief goods at the distribution center, the vehicle waits at the distribution center until new relief goods arrive.

\subsection{Delivery of information by epidemic transmission}

In this paper, the communication nodes are the transport vehicles with communication devices, the shelters with communication devices, and the distribution center with a communication device. Suppose that a communication device consists of a computer, a storage, and a wireless device with capability of direct communication such as the ad hoc mode of the wireless LAN. Each communication device has the function of epidemic transmission.

An example of the system structure is shown in Fig. [1. Suppose that the communication nodes have to communicate directly via wireless links because communication infrastructures such as cellular systems are disabled by the disaster. We assume that communication nodes exchange information if they are at the same place, such as the shelters and the dis- 


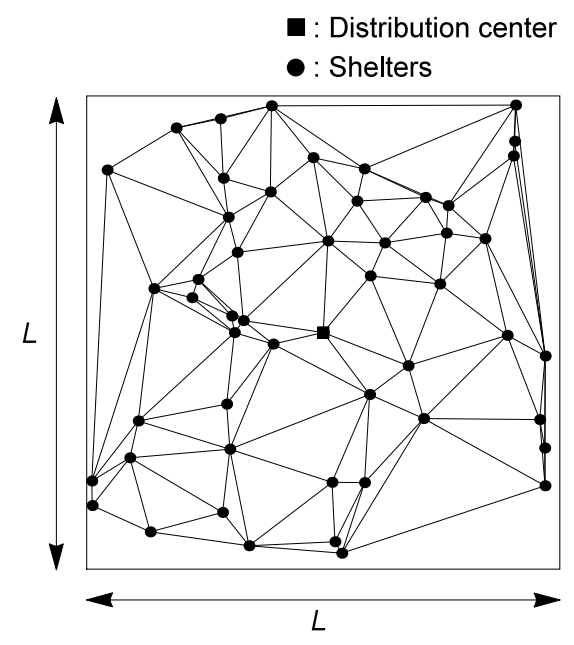

Figure 1: Road network.

tribution center, and that the transport vehicles do not exchange information on the paths to clearly show the effects of additional waiting time on performance. Using the epidemic transmission function, a vehicle collects information from the shelters and delivers it to other shelters while moving along the path. The vehicle repeatedly stops at the distribution center to pick up new relief goods. At the same time, it transfers information collected from shelters to other vehicles stopping at the distribution center. Then the information is propagated over other traveling paths. If a transport vehicle does not make any contact with other transport vehicles, it transfers information to a communication device installed at the distribution center, and the communication device passes the information to transport vehicles arriving at the distribution center later.

The main objective of this paper is to develop epidemic transmission in disaster situations; therefore, the information delivery sometimes involves a long delay because of the nature of epidemic transmission. Because it is difficult to deliver a very urgent information through epidemic transmission, we consider information that is not so urgent. We consider information on the status of shelters such as the list of evacuees in the shelter and a request of relief goods. Information is periodically generated at each shelter. If no vehicle arrives at a shelter before new information is generated at it, the old information is updated with the new information.

To enhance the information exchange performance, we increase the opportunity for a vehicle to meet other vehicles at the distribution center. We keep a vehicle at time period $t_{w}$ at the distribution center after the relief goods are completely loaded. If all $m$ vehicles gather at the distribution center and are loaded within $t_{w}$, then $m$ vehicles leave the distribution center. This additional staying time is expected to improve the performance of the information exchange; however, at the same time, since the shelter might be kept waiting for a longer time for relief goods, we analyze this trade-off relationship in the next section. 


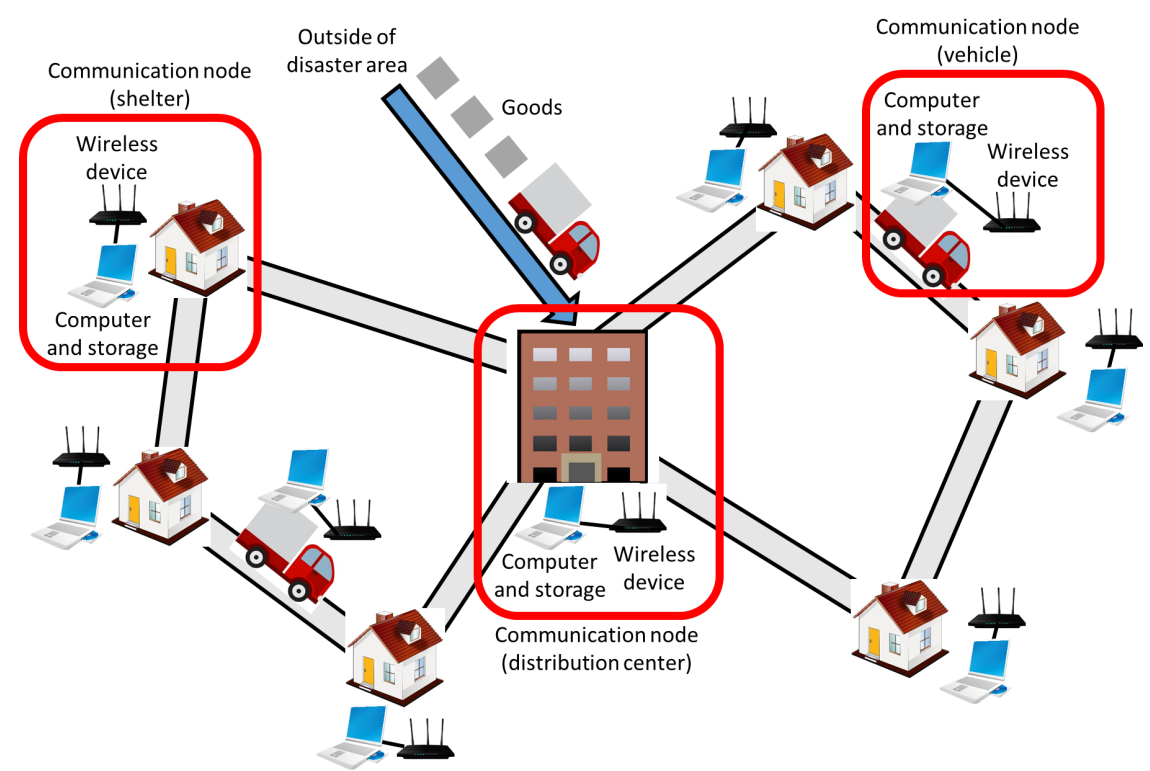

Figure 2: System structure.

\subsection{Performance evaluation}

For our performance analysis, we used three metrics:

- $E\left(T_{G}\right)$ : the mean time interval of the arrival of relief goods at a shelter.

- $E\left(T_{I}\right)$ : the mean time interval of the arrival of information at a shelter.

- $E\left(T_{D}\right)$ : the mean delay of the arrival of information, which is the interval from the time when information is generated to its arrival at a shelter.

To evaluate the information sharing performance, we use $E\left(T_{I}\right)$ and $E\left(T_{D}\right) . E\left(T_{I}\right)$ denotes how frequently information is updated at each shelter, and $E\left(T_{D}\right)$ is the delay of the arrival of new information. For the performance evaluation of the relief goods delivery, we use only $E\left(T_{G}\right)$, which denotes how frequently relief goods arrive at each shelter, and ignore the delay of such new relief goods as $E\left(T_{D}\right)$. This is because at shelters, people who are interested in how frequently relief goods are provided are not interested in their arrival at the distribution center. We consider the relations of $t_{w}$ to $E\left(T_{G}\right), E\left(T_{I}\right)$, and $E\left(T_{D}\right)$ to study its positive and negative effects on performance.

In our performance evaluations, we consider two extreme cases:

- Case 1: $t_{w}=0$.

- Case $2: t_{w}$ is sufficiently large.

In Case 1, a transport vehicle never waits for other transport vehicles at the distribution center. Therefore, a transport vehicle usually gets only information that is left at the distribution center by other nodes that have already departed to make their deliveries. 
We say that two transport vehicles are synchronized if they meet at the distribution center and exchange information. In contrast to Case 1 , we assume that additional waiting time $t_{w}$ is sufficiently large to consider a case where a transport vehicle can be synchronized with all the other transport vehicles at the distribution center while the transport vehicle stays at the distribution center. $t_{i}$ is the time needed for one trip of an $i$ th traveling path, $t_{\max }=\max _{i} t_{i}$, $t_{\min }=\min _{i} t_{i}$, and $t_{d i f}=t_{\max }-t_{\min }$. A transport vehicle can meet all the other transport vehicles at the distribution center if $t_{w} \geq t_{\text {dif }}$. This is explained as follows: All transport vehicles leave the distribution center in Case 2 and a transport vehicle with $t_{\min }$ arrives at the distribution center the earliest and the transport vehicle with $t_{\max }$ arrives the latest at the distribution center. Then the transport vehicle with $t_{\min }$ can meet the transport vehicle with $t_{\max }$ at the distribution center if it waits for $t_{d i f}$.

As assumed in Section [2.2, if all the transport vehicles arrive at the distribution center and are ready to leave after loading the relief goods, they leave the distribution center even if the waiting times of some of the vehicles are less than $t_{w}$. Because all the transport vehicles always gather at the distribution center in Case 2, all of them always leave at the same time in Case 2. Hence, a transport vehicle in Case 2 is expected to get newer information, as in Case 1.

We compute the delivery performances of the relief goods and the delivery of the information in cases other than Cases 1 and 2 by computer simulation and characterize their performances by comparing them with the extreme behaviors in Cases 1 and 2.

\section{Assumptions and parameters for simulation}

In this paper, we assume that a service area is a square of side $L$. A distribution center is located at the center of the service area. $n$ is the number of shelters, and these are distributed randomly. Transport vehicles move along a road network, which connects the shelters (Fig. W). Suppose that the roads in the road network do not intersect. Let $m$ be the number of transport vehicles that carry information with relief goods on $m$ different traveling paths.

A transport vehicle chooses one of the $m$ paths, and $m$ vehicles move along different paths at velocity $v$. We use the Route First-Cluster Second (RFCS) method [ए]] to construct $m$ traveling paths. RFCS is a simple and effective algorithm to decide the transportation schedule of relief goods. The following are the details of the RFCS method:

1. For all the pairs of shelters $\left(a_{i}, a_{j}\right)$, compute the total distance of the shortest path between them, denoted by $x_{i, j}$, using such a shortest path algorithm as the Dijkstra algorithm [18].

2. Solve the traveling salesman problem with an approximate method, such as the previously introduced methods $[\mathbb{[}]$ and obtain a circuit that contains all $n$ shelters. In this step, $x_{i, j}$ is the cost of a link between $a_{i}$ and $a_{j}$ because not all the shelter pairs always have a road that directly connects them. Let $a_{1}, a_{2}, \cdots, a_{n}$ be the shelters in the order of appearance in the obtained circuit. For generality, we also denote by $a_{0}$ the distribution center. Figure 3$]$ shows the obtained circuit for the road network in Fig. 四. 
3. Divide the above circuit into $m$ small circuits by defining the cost for each possible small circuit. Let $c_{i, j}$ be the small circuit that passes $a_{0}, a_{i+1}, a_{i+2}, \cdots, a_{j}$, and $a_{0}$ in this order. We define the cost of $c_{i, j}$, denoted by $y_{i, j}$, as follows:

$$
y_{i, j}= \begin{cases}\infty, & i \geq j, \\ \infty, & j-i>\left\lceil\frac{n}{m}\right\rceil, \\ x_{0, i+1}+\sum_{k=i+1}^{j-1} x_{k, k+1}+x_{j, 0}+\text { const, } & \text { otherwise. }\end{cases}
$$

Note that the second constraint is to avoid dropping the number of generated small circuits less than $m$, and const is a constant for avoiding a number of generated small circuits that exceeds $m$. In this paper, we set const to 1000 and constructed a weighted graph where a node corresponds to a shelter and the cost of a link from $a_{i}$ to $a_{j}$ corresponds to $y_{i, j}$. In this weighted graph, we compute the shortest path from $a_{0}$ to $a_{n}$ using a shortest path algorithm, such as the Dijkstra algorithm [ए8]. If the shortest path consists of $a_{0}, a_{i_{1}}, a_{i_{2}}, \cdots, a_{i_{m-1}}$, and $a_{n}$, then we obtain $m$ small circuits $c_{0, i_{1}}$, $c_{i_{1}, i_{2}}, c_{i_{2}, i_{3}}, \cdots, c_{i_{m-2}, i_{m-1}}, c_{i_{m-1}, n}$. Figure 目 shows the small circuits obtained by dividing the circuit of Fig. [], where $m=5$.

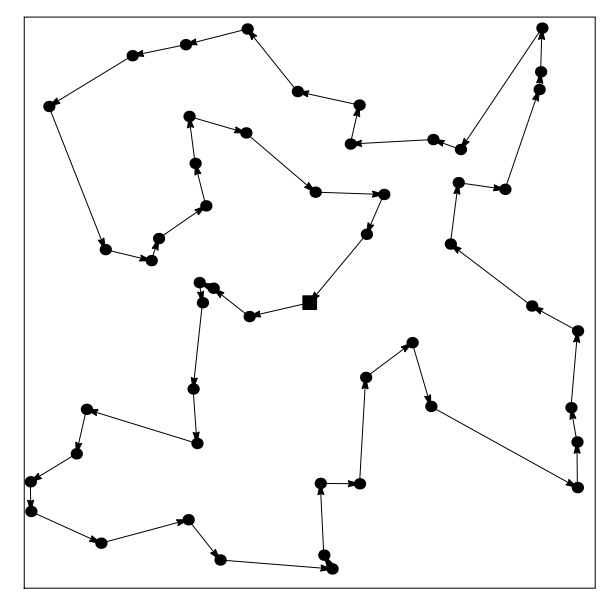

Figure 3: Circuit that contains all $n$ shelters for the road network in Fig. 1 .

The stochastic process of the arrival of relief goods at the distribution center obeys a Poisson process of intensity $\lambda_{G}$. The stochastic process of the arrival of new information at a shelter also obeys a Poisson process of intensity $\lambda_{I}$.

In the computer simulations, all $m$ vehicles are at the distribution center at the initial time, and these vehicles start to deliver relief goods to the shelters after the relief goods first arrive at the distribution center. The movements of each vehicle and the exchange of information are based on the assumptions in Section 2 . We observed the time when each relief good and each bit of information were delivered to each shelter until 1000 relief goods are delivered to all the shelters and computed $E\left(T_{G}\right), E\left(T_{I}\right)$, and $E\left(T_{D}\right)$ from these times. 


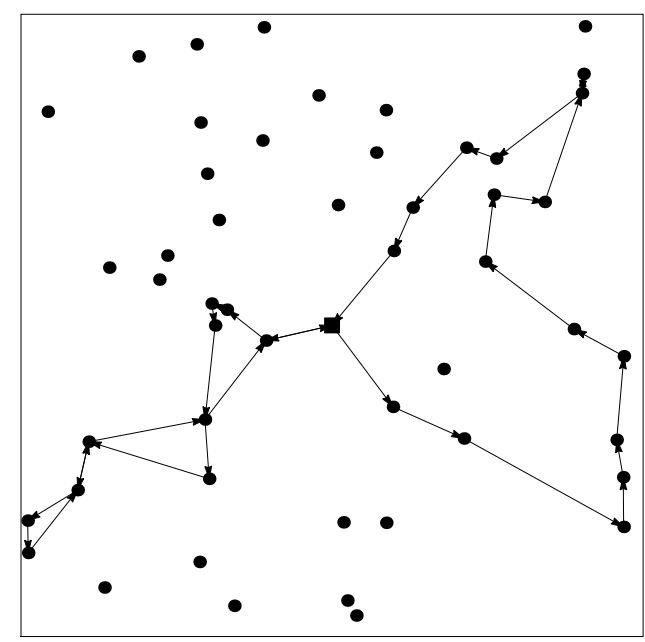

(a) 1st and 3rd paths.

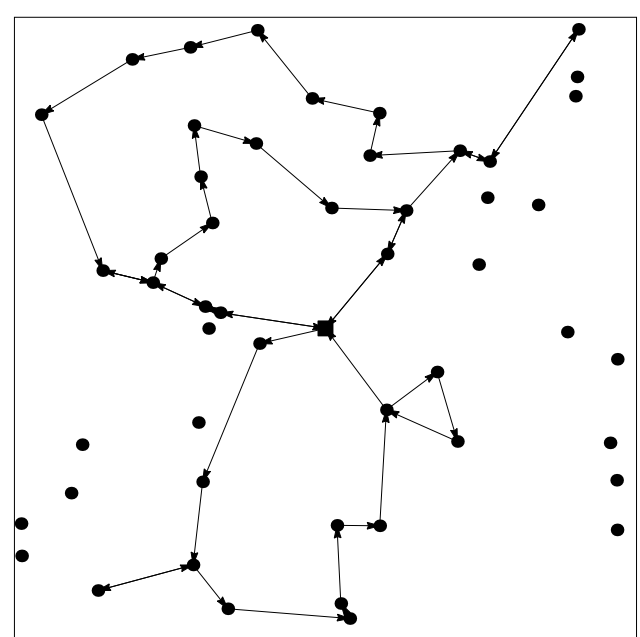

(b) 2nd, 4th, and 5th paths.

Figure 4: Traveling paths for road network in Fig. W obtained by dividing circuit of Fig. [] into five traveling paths.

In computer simulations, we used the following values of the parameters summarized in Table W: $t_{1}=7.10$ hour, $t_{2}=7.50$ hour, $t_{3}=7.65$ hour, $t_{4}=7.96$ hour, $t_{5}=6.87$ hour, $t_{\min }=t_{5}=6.87$ hour, $t_{\max }=t_{4}=7.96$ hour, and $t_{\text {dif }}=1.09$ hour $=65.4 \mathrm{~min}$.

Table 1: Values of parameters used in simulation.

\begin{tabular}{c|c}
\hline Parameter & Value \\
\hline Size of service area, $L$ & $10 \mathrm{~km}$ \\
Number of shelters, $n$ & 50 \\
Number of transport vehicles, $m$ & 5 \\
Velocity of a transport vehicle, $v$ & $10 \mathrm{~km} / \mathrm{hour}$ \\
Loading and unloading time at one shelter, $t_{\text {stay }}$ & $30 \mathrm{~min}$ \\
Arrival rate of relief goods at distribution center, $\lambda_{G}$ & 0.1 hour $^{-1}, 0.25$ hour $^{-1}$ \\
Arrival rate of information at each shelter, $\lambda_{I}$ & 0.25 hour $^{-1}$ \\
\hline
\end{tabular}

\section{Simulation results and discussions}

\subsection{Results for low arrival rate of relief goods}

In this section, we show the simulation results of $E\left(T_{G}\right), E\left(T_{I}\right)$, and $E\left(T_{D}\right)$ and discuss how they are affected by additional waiting time $t_{w}$. Figure $\square$ shows the results for $\lambda_{G}=0.1$ hour $^{-1}$. The arrival rate of the relief goods at the distribution center is low. From Fig. [], $E\left(T_{G}\right), E\left(T_{I}\right)$, and $E\left(T_{D}\right)$ are only slightly affected by $t_{w}$. This tendency suggests that waiting 
for other vehicles at the distribution center has little meaning if the arrival rate of the relief goods is low.

To consider the reason for the above tendency, we show another results obtained in the computer simulations: the mean time spent for one trip for $i$ th vehicle, denoted by $E\left(T_{\text {trip }, i}\right)$, and the mean number of vehicles that $i$ th vehicle meets at the distribution center, denoted

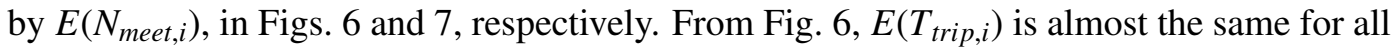
$i$, and this value does not greatly change if $t_{w}$ increases. This is because for a low $\lambda_{G}$, each vehicle often waits for new relief goods to arrive at the distribution center longer time than $t_{w}$. In fact, the value of $E\left(T_{\text {trip, },}\right)$ in this case is around $\frac{1}{\lambda_{G}}=10$ hour, which is the mean time interval of the arrival of relief goods at the distribution center. For this reason, it is considered that $E\left(T_{G}\right)$ and $E\left(T_{I}\right)$ were only slightly affected by $t_{w}$ if $\lambda_{G}$ is low.

From Fig. $\mathbb{Q}, E\left(N_{\text {meet }, i}\right)$ gradually increases with $t_{w}$ and becomes the maximum value if $t_{w}=t_{\text {dif }}=65.4 \mathrm{~min}$. Note that the maximum value of $E\left(N_{\text {meet }, i}\right)$ is 4 because the number of transport vehicles is 5. However, $E\left(N_{\text {meet }, i}\right)$ is not so small even for $t_{w}=0$ because the vehicles often stay at the distribution center for a long time to wait for new relief goods if $\lambda_{G}$ is low as mentioned, and they meet each other during waiting for new relief goods. For this reason, it is considered that $E\left(T_{D}\right)$ was also only slightly affected by $t_{w}$ if $\lambda_{G}$ is low.

\subsection{Results for high arrival rate of relief goods}

Figure 8 shows the results for a large arrival rate of relief goods and $\lambda_{G}=0.25$ hour $^{-1}$. $E\left(T_{G}\right), E\left(T_{I}\right)$, and $E\left(T_{D}\right)$ increase as $t_{w}$ increases and rapidly decrease if $t_{w}$ exceeds $t_{d i f}$. If $t_{w} \geq t_{d i f}$, then the system is identical to Case 2, in which all vehicles are synchronized completely; therefore, the performance is almost the same. $t_{d i f}$ is sufficient for the additional waiting time in Case 2.

To consider the reason for the above tendency, we show the results of $E\left(T_{\text {trip }, i}\right)$ and $E\left(N_{\text {meet }, i}\right)$ in Figs. $⿴$ and $\mathbf{0}$, respectively. From Fig. Q, $E\left(T_{\text {trip }, i}\right)$ increases with $t_{w}$ if $t_{w}<65.4$ min. This is because for a high $\lambda_{G}$, vehicles usually wait just $t_{w}$ at the distribution center because they rarely wait for new relief goods to arrive at the distribution center. In fact, the value of $E\left(T_{\text {trip }, i}\right)$ for $t_{w}=0$ is around $t_{i}$ for all $i$. However, if $t_{w}=t_{\text {dif }}=65.4 \mathrm{~min}$, then $E\left(T_{\text {trip }, i}\right)$ rapidly decreases. This is because when $t_{w} \geq t_{d i f}$, all the vehicles are completely synchronized, and then some vehicles leave the distribution center without waiting $t_{w}$. For this reason, it is considered that $E\left(T_{G}\right)$ and $E\left(T_{I}\right)$ increase as $t_{w}$ increases if $t_{w}<t_{d i f}$, and rapidly decrease if $t_{w}$ exceeds $t_{d i f}$.

From Fig. 미, $E\left(N_{m e e t, i}\right)$ gradually increases with $t_{w}$ and becomes the maximum value if $t_{w}=t_{\text {dif }}=65.4 \mathrm{~min}$ in the same manner as for $\lambda_{G}=0.1$ hour $^{-1}$. However, unlike the results for $\lambda_{G}=0.1$ hour $^{-1}, E\left(N_{\text {meet }, i}\right)$ for $t_{w}<t_{d i f}$ is small. For this reason, when $t_{w}$ exceeds $t_{d i f}$, $E\left(T_{D}\right)$ rapidly decreases because the information sharing among vehicles becomes effective rapidly.

The above results indicate that if $\lambda_{G}$ is large, additional waiting time $t_{w}$ should be 0 or $t_{d i f}$ because $E\left(T_{G}\right), E\left(T_{I}\right)$, and $E\left(T_{D}\right)$ increase for $0<t_{w}<t_{d i f}$. Next we compared $E\left(T_{G}\right)$ for $t_{w}=0$ and $t_{w} \geq t_{d i f}$ to find the best value of $t_{w}$. E(T $\left.T_{G}\right)$ for $t_{w} \geq t_{\text {dif }}$ is slightly larger than that for $t_{w}=0$. This is because the delivery of relief goods is obviously fastest if no vehicle ever waits for other vehicles. For $E\left(T_{I}\right)$, we have the same tendency as $E\left(T_{G}\right)$. The 


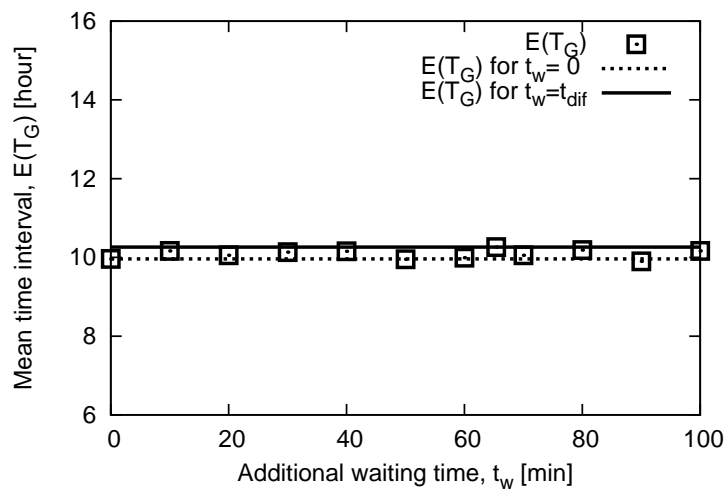

(a) $E\left(T_{G}\right)$.

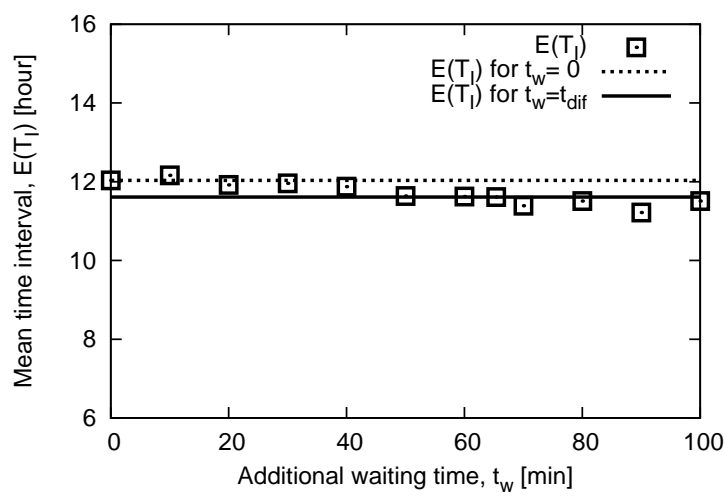

(b) $E\left(T_{I}\right)$.

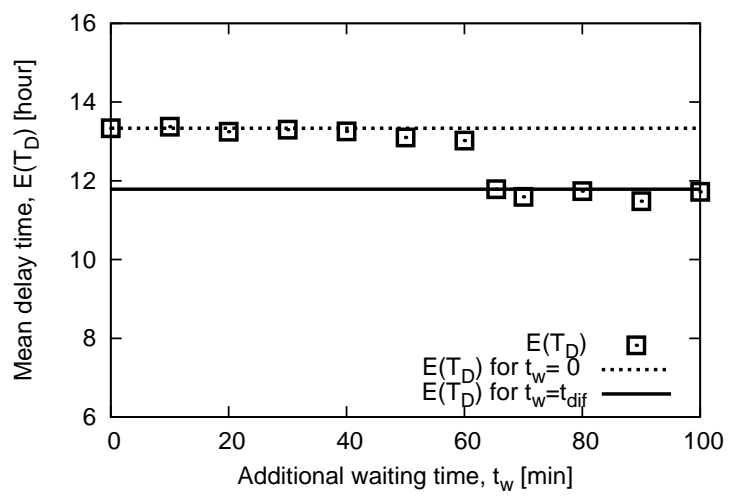

(c) $E\left(T_{D}\right)$.

Figure 5: Results for $\lambda_{G}=0.1$ hour $^{-1}$. 


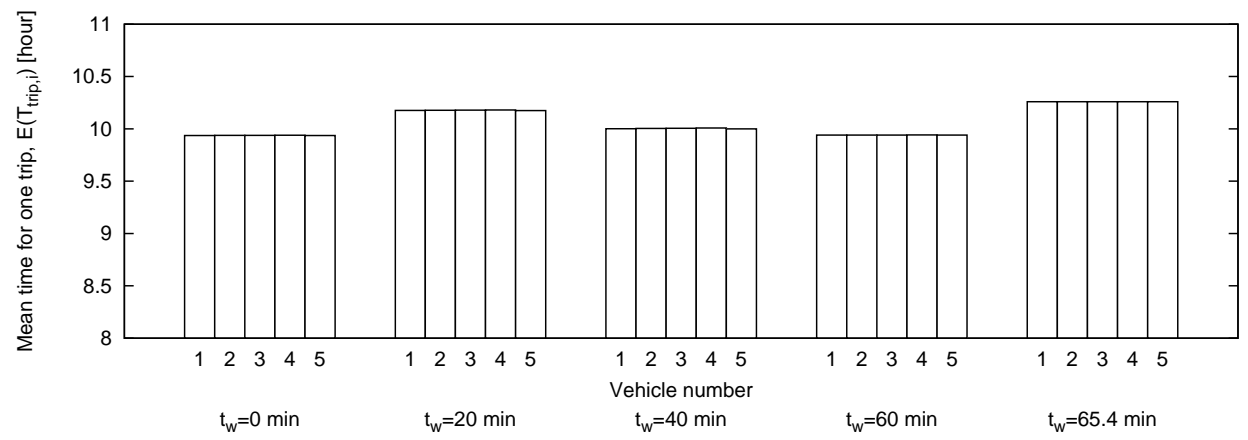

Figure 6: Mean time spent for one trip for each vehicle in the computer simulations, where $\lambda_{G}=0.1$ hour $^{-1}$.

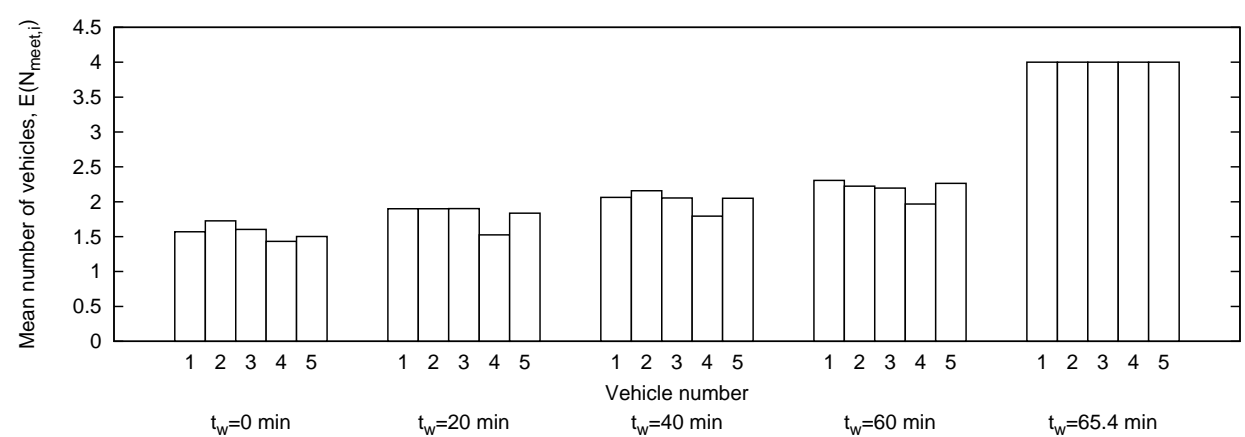

Figure 7: Mean number of vehicles that each vehicle meets at the distribution center in the computer simulations, where $\lambda_{G}=0.1$ hour $^{-1}$. 


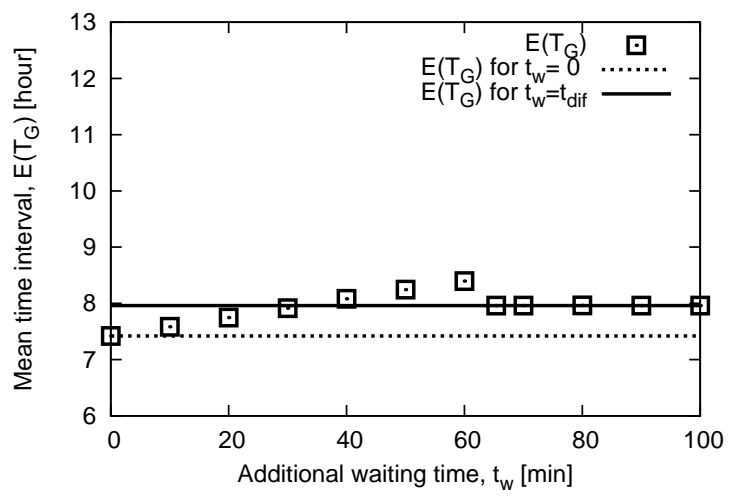

(a) $E\left(T_{G}\right)$.

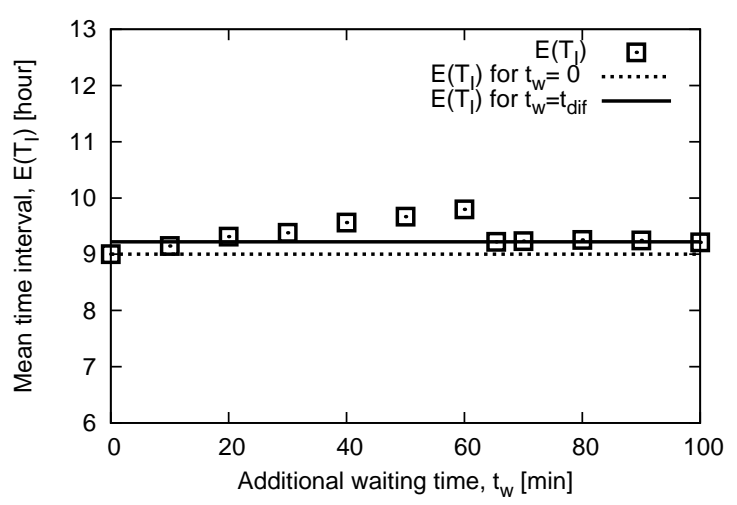

(b) $E\left(T_{I}\right)$.

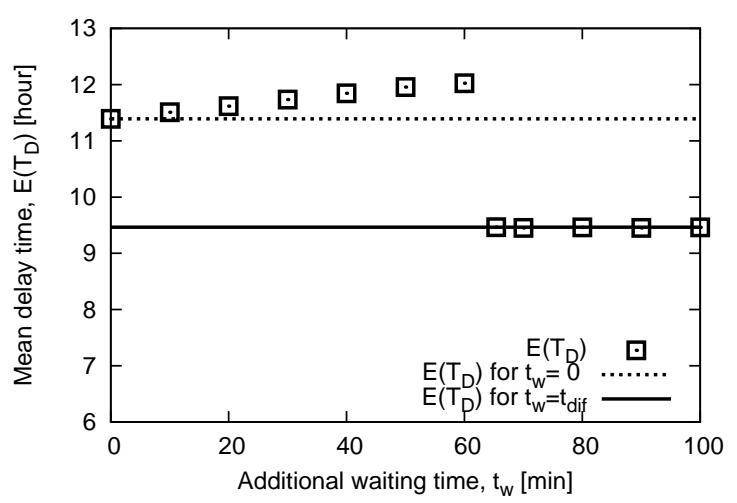

(c) $E\left(T_{D}\right)$.

Figure 8: Results for $\lambda_{G}=0.25$ hour $^{-1}$. 


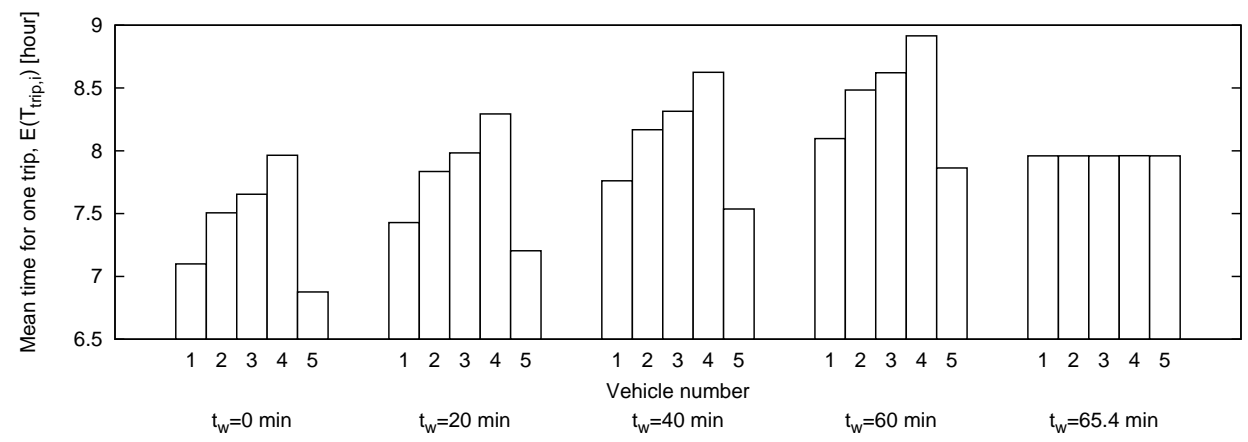

Figure 9: Mean time spent for one trip for each vehicle in the computer simulations, where $\lambda_{G}=0.25$ hour $^{-1}$.

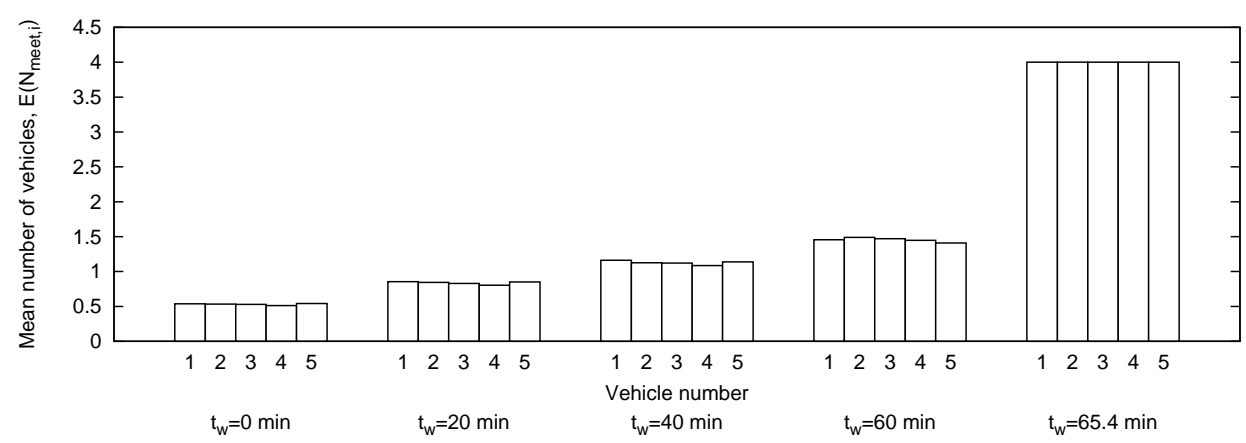

Figure 10: Mean number of vehicles that each vehicle meets at the distribution center in the computer simulations, where $\lambda_{G}=0.25$ hour $^{-1}$. 
best value is $t_{w}=0$ for $E\left(T_{G}\right)$ and $E\left(T_{I}\right)$.

Next, we compared $E\left(T_{D}\right)$ for $t_{w}=0$ and for $t_{w} \geq t_{\text {dif }}$. $E\left(T_{D}\right)$ for $t_{w} \geq t_{d i f}$ is much smaller than $E\left(T_{D}\right)$ for $t_{w}=0$. The best value is $t_{w}=t_{\text {dif }}$ for $E\left(T_{D}\right)$ because all the transport vehicles can completely share information because of complete synchronization.

As mentioned above, we evaluated two types of performance metrics: the mean time interval between adjacent arrivals at each shelter for the performances of relief goods delivery and information sharing (i.e., $E\left(T_{G}\right)$ and $E\left(T_{I}\right)$ ) as well as the mean delay time for the information sharing performance (i.e., $E\left(T_{D}\right)$ ). The first metric does not vary much as $t_{w}$ changes. However, the second type changes more rapidly than the first. From this viewpoint, although additional waiting time did not affect the performance much if we consider the mean time interval between adjacent arrivals to be a metric, waiting a sufficiently long time so that all the vehicles are completely synchronized greatly improved the performance if we consider the mean delay time to be a metric.

\subsection{Results for different distribution patterns of shelters}

Next, we evaluated $E\left(T_{G}\right), E\left(T_{I}\right)$, and $E\left(T_{D}\right)$ for different distribution patterns of shelters. Patterns 1 to 4 are shown in Fig. $\square$. Pattern 1 has the same pattern as the above results. Figures [2] and [13 show $E\left(T_{G}\right), E\left(T_{I}\right)$, and $E\left(T_{D}\right)$ for Patterns 1 to 4 , where $\lambda_{G}=0.1$ hour $^{-1}$ and $\lambda_{G}=0.25$ hour $^{-1}$, respectively. The other parameters are the same as the above results. These figures show a similar tendency of $E\left(T_{G}\right), E\left(T_{I}\right)$, and $E\left(T_{D}\right)$ even for different distribution patterns of shelters. This indicates that we found the same results in Figs. 5 and 8 even for different distribution patterns of shelters if the size of the disaster area and the number of shelters are given.

\subsection{Effects of arrival rate of relief goods and number of vehicles}

In this paper, we consider the information that is not so urgent because epidemic transmission is applied. Different kinds of information have different degrees of urgency. If we can reduce $E\left(T_{I}\right)$ and $E\left(T_{D}\right)$, we may use the epidemic transmission in more urgent situations. To explain the relation between such different levels of urgency and $E\left(T_{I}\right)$ and $E\left(T_{D}\right)$, we consider four patterns that are different in the arrival rate of relief goods $\lambda_{G}$ and the number of vehicles $m$ as follows. We again consider Fig. 미, where $\lambda_{G}=0.1$ hour $^{-1}$ and $m=5$, and Fig. [3], where $\lambda_{G}=0.25$ hour $^{-1}$ and $m=5$, and show Fig. [4] for $\lambda_{G}=0.1$ hour $^{-1}$ and $m=10$ and Fig. 15 for $\lambda_{G}=0.25$ hour $^{-1}$ and $m=10$. Consider Figs. $\square 2$ and [4]. In these figures, $\lambda_{G}$ is small and $m$ is different. In this case, $E\left(T_{I}\right)$ and $E\left(T_{D}\right)$ do not decrease so much even if $m$ increases. This is because $E\left(T_{I}\right)$ and $E\left(T_{D}\right)$ greatly depend on the waiting time for relief goods at the distribution center because of a small $\lambda_{G}$ and the effect of increase of $m$ is eliminated. Next, consider Figs. [3] and [55. In these figures, $\lambda_{G}$ is large and $m$ is different. In this case, $E\left(T_{I}\right)$ and $E\left(T_{D}\right)$ decrease as $m$ increases. It is considered that increase of $m$ effectively decreases $E\left(T_{I}\right)$ and $E\left(T_{D}\right)$ if the waiting time for relief goods at the distribution center decreases due to a large $\lambda_{G}$.

As mentioned above, if $\lambda_{G}$ is large, $E\left(T_{I}\right)$ and $E\left(T_{D}\right)$ greatly decrease if $m$ increases. On the other hand, if $\lambda_{G}$ is small, it is sometimes difficult to reduce $E\left(T_{I}\right)$ and $E\left(T_{D}\right)$ by 


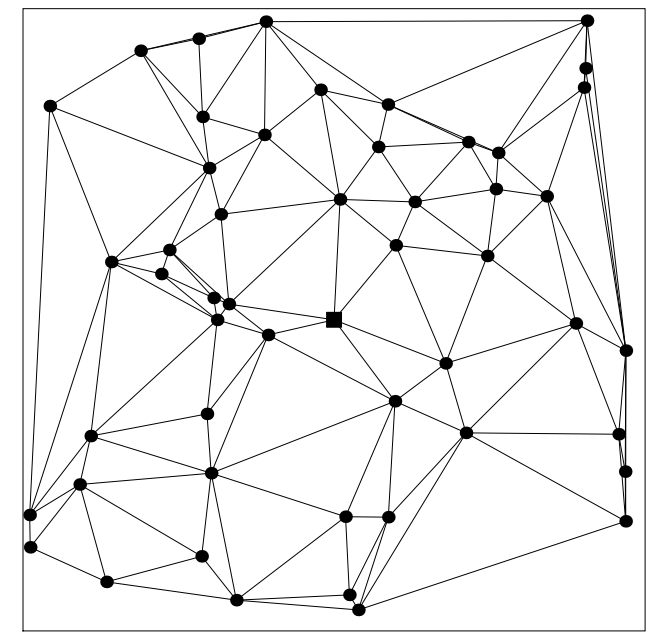

(a) Pattern 1.

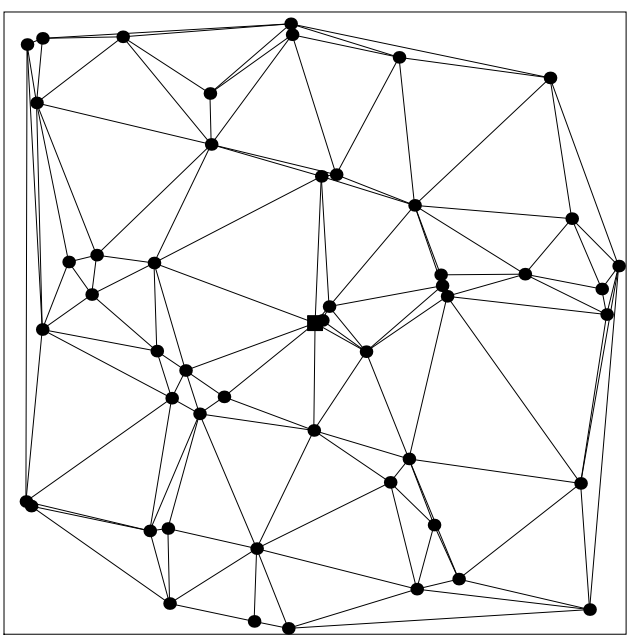

(c) Pattern 3.

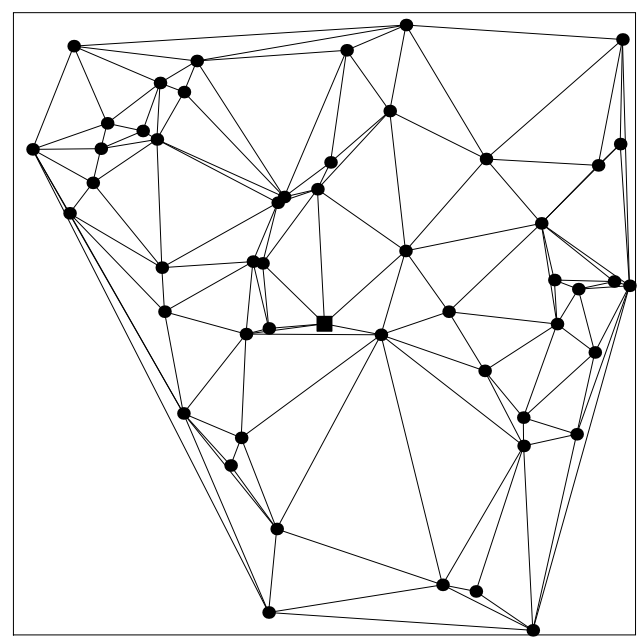

(b) Pattern 2.

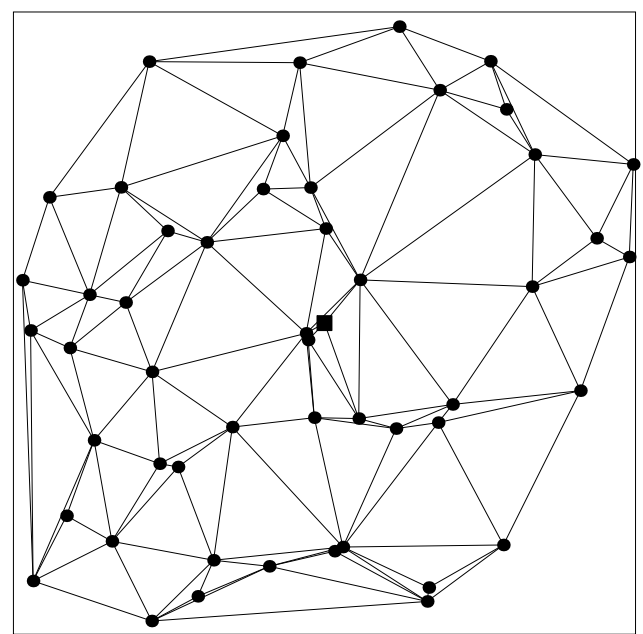

(d) Pattern 4.

Figure 11: Four distribution patterns of shelters. 


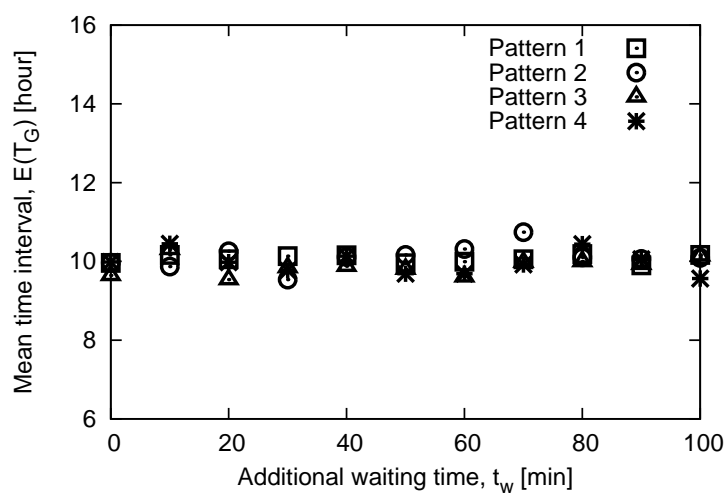

(a) $E\left(T_{G}\right)$.

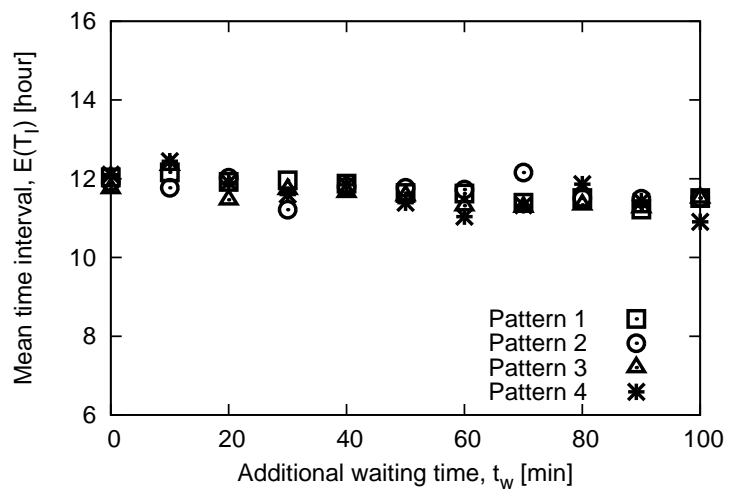

(b) $E\left(T_{I}\right)$.

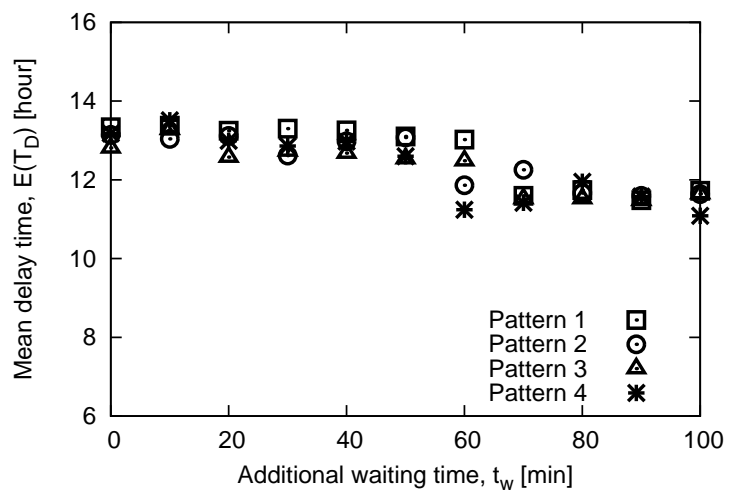

(c) $E\left(T_{D}\right)$.

Figure 12: Results for Patterns 1 to 4 , where $m=5$ and $\lambda_{G}=0.1$ hour $^{-1}$. 


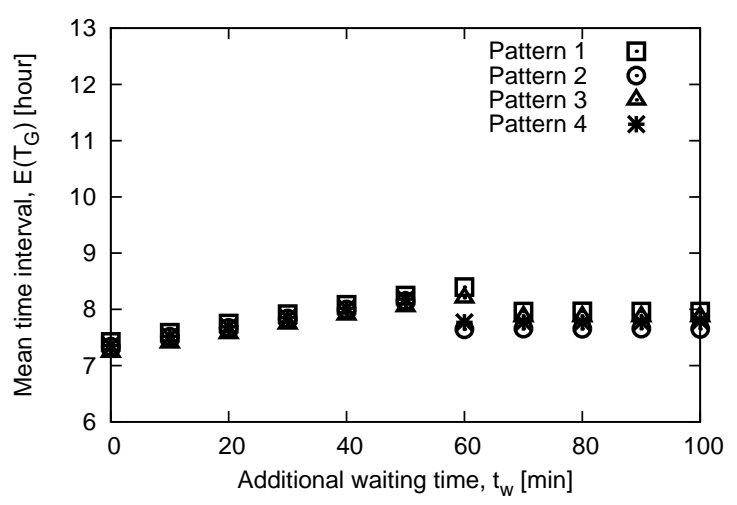

(a) $E\left(T_{G}\right)$.

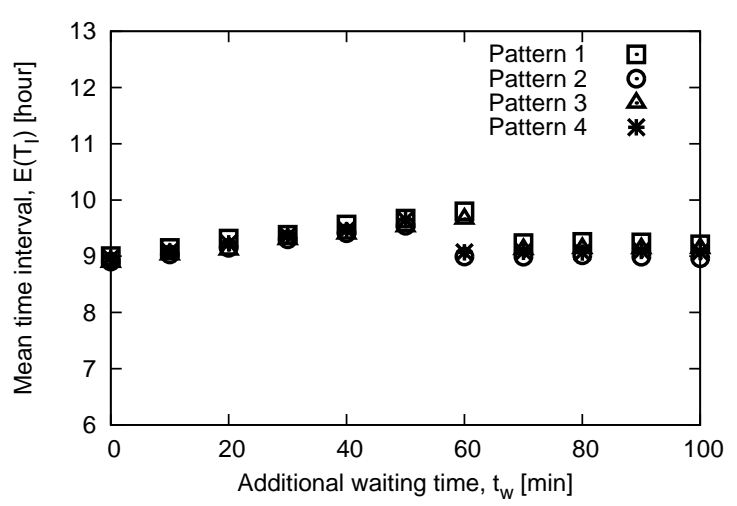

(b) $E\left(T_{I}\right)$.

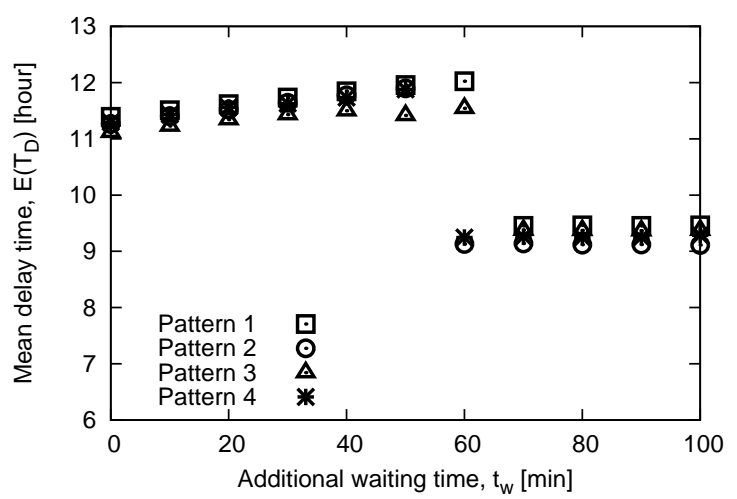

(c) $E\left(T_{D}\right)$

Figure 13: Results for Patterns 1 to 4 , where $m=5$ and $\lambda_{G}=0.25$ hour $^{-1}$. 
increasing $m$. Therefore, if $\lambda_{G}$ is large, we can apply epidemic transmission with transport vehicles to more urgent purposes by increasing transport vehicles. If $\lambda_{G}$ is small, however, increasing transport vehicles is not effective on applying epidemic transmission to more urgent purposes.

\section{Conclusion}

In this paper, we considered information exchange among isolated shelters using epidemic transmission during large-scale disasters. As mobile nodes for epidemic transmission, we used transport vehicles that leave a distribution center to deliver relief goods to shelters. To enhance the information sharing performance among shelters in such situations, our method forced transport vehicles to wait for the arrival of other transport vehicles at the distribution center and analyzed how the additional waiting time affected the delay of relief goods and the time needed for information sharing by computer simulation. Our simulation results showed that if the arrival rate of relief goods is low, then waiting for other vehicles at the distribution center has little meaning. If the arrival rate of relief goods is high, although additional waiting time does not affect the performance much if we consider the mean time interval between adjacent arrivals to be a metric, waiting a sufficiently long time greatly improves the performance if we consider the mean delay time to be a metric.

\section{Acknowledgments}

This work is partially supported by JSPS KAKENHI Grant Number 25420360. The authors thank Yuta Otani of the Graduate School of Science and Technology, Niigata University for his help. The authors thank anonymous reviewers for their valuable comments.

\section{References}

[1] B. V. D. Walle, M. Turoff, and S. R. Hiltz, Information Systems for Emergency Management (Advances in Management Information Systems), M.E. Sharpe, 2010.

[2] V. Jones, G. Karagiannis, and S. Heemstra de Groot, "Ad hoc networking and ambient intelligence to support future disaster response," Proc. IEEE ASWN05, pp. 137-146, 2005.

[3] Special Section on Schemes and Controllability of Advanced Information and Communication Networks for Disasters, The Journal of IEICE, vol. 89, no. 9, Sept. 2006. (in Japanese)

[4] W. Guo and X. Huang, "On coverage and capacity for disaster area wireless networks using mobile relays," EURASIP Journal on Wireless Communications and Networking, vol. 2009, pp. 1-17, Jan. 2009.

[5] R. Bruno, M. Conti, A. Passarella, "Opportunistic networking overlays for ICT services in crisis management,” Proc. ISCRAM 2008, pp. 689-701, May 2008. 


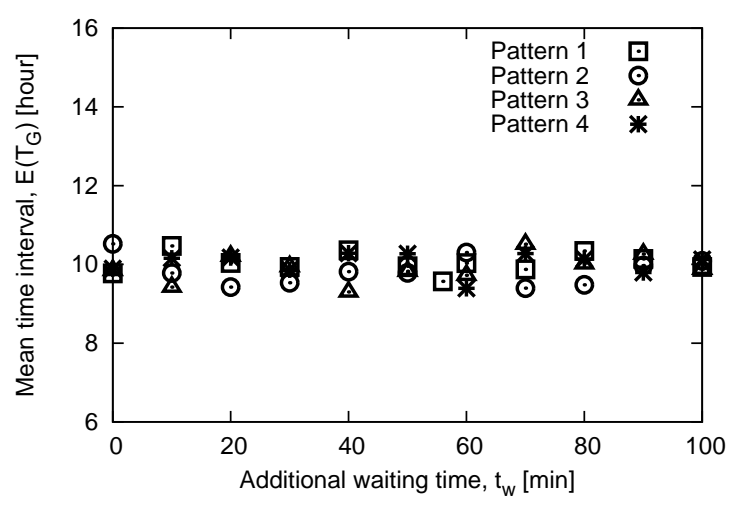

(a) $E\left(T_{G}\right)$.

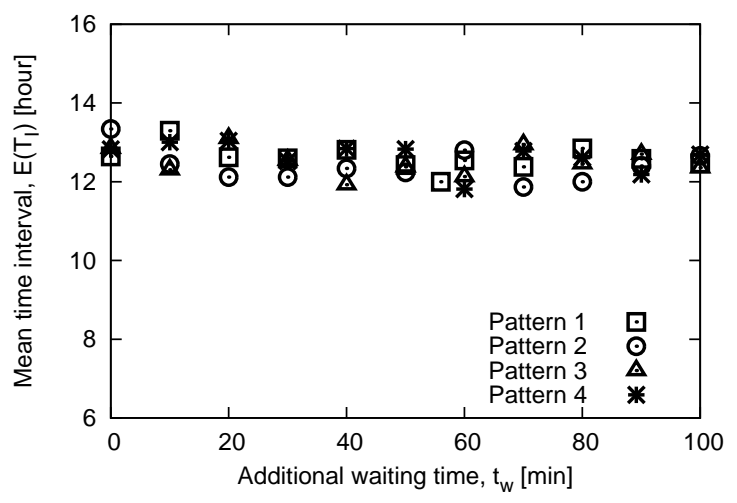

(b) $E\left(T_{I}\right)$.

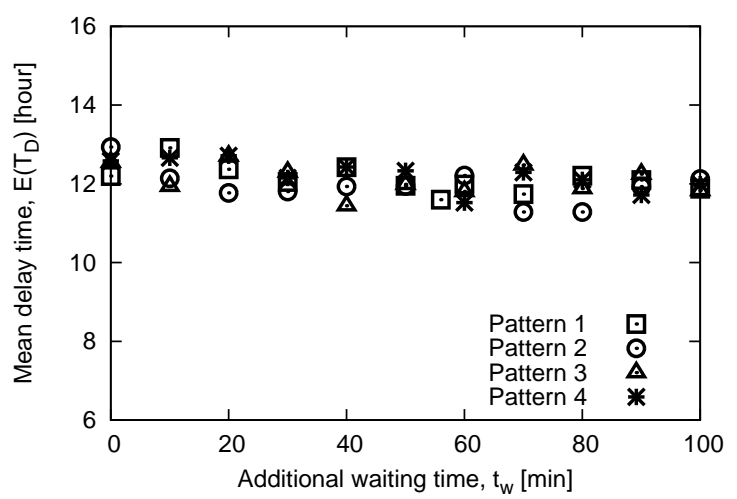

(c) $E\left(T_{D}\right)$.

Figure 14: Results for Patterns 1 to 4 , where $m=10$ and $\lambda_{G}=0.1$ hour $^{-1}$. 


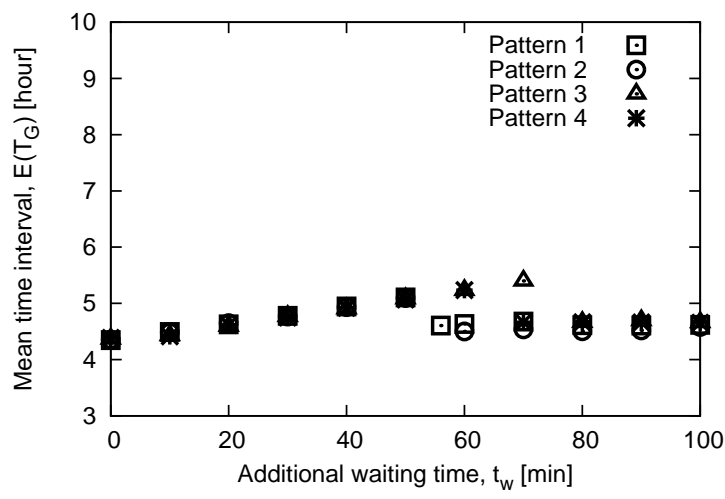

(a) $E\left(T_{G}\right)$.

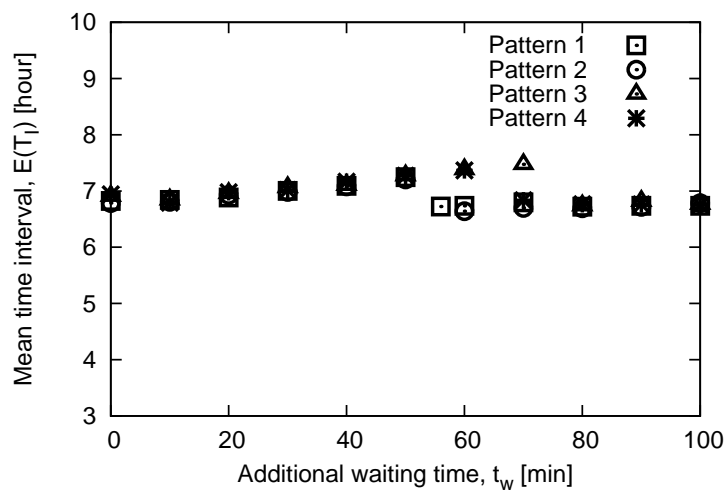

(b) $E\left(T_{I}\right)$

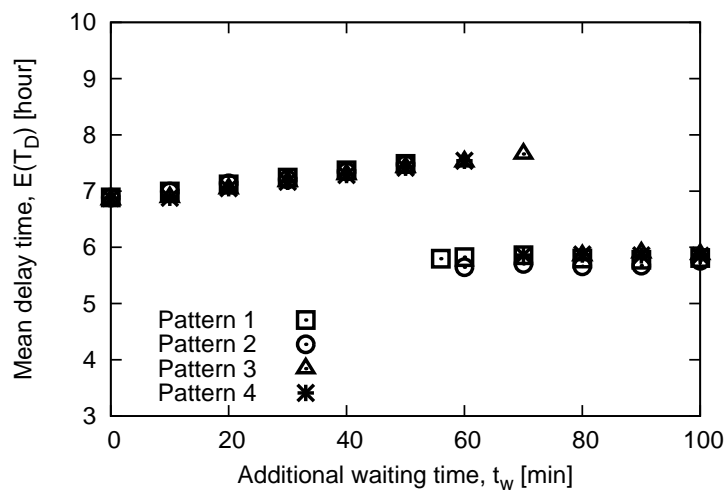

(c) $E\left(T_{D}\right)$.

Figure 15: Results for Patterns 1 to 4 , where $m=10$ and $\lambda_{G}=0.25$ hour $^{-1}$. 
[6] A. Vahdat and D. Becker, "Epidemic routing for partially-connected ad hoc networks," Technical Report CS-2000-06, Duke University, 2000.

[7] K. Fall, "A delay-tolerant network architecture for challenged internets," Intel Research Technical Report, IRB-TR-03-003, Feb. 2003.

[8] F. Warthman, Delay tolerant networks (DTNs): A tutorial, DTN Research Group, Internet Draft, 2003.

[9] C.D.T.W. Gandy, L.R. Foulds, "The vehicle scheduling problem: a survey," New Zealand Operational Research, vol. 9, no. 2, pp. 73-92, 1981.

[10] J.E. Beasley, "Route First Cluster Second Methods for Vehicle Routing," OMEGA International Journal of Management Science, vol. 11, no. 4, pp. 403-408, 1983.

[11] M. Horner, J. Downs, "Testing a flexible geographic information system-based network flow model for routing hurricane disaster relief goods," Transportation Research Record, vol. 2022, pp. 47-53, 2007.

[12] Y. M. Lee, S. Ghosh, M. Ettl, "Simulating distribution of emergency relief supplies for disaster response operations," Proc. IEEE WSC 2009, pp. 2797-2808, Dec. 2009.

[13] M. J. Widener and M. W. Horner, "A hierarchical approach to modeling hurricane disaster relief goods distribution," Journal of Transport Geography, vol. 19, issue 4, pp. 821-828, July 2011.

[14] Y. Han, X. Guan, L. Shi, "Optimization based method for supply location selection and routing in large scale emergency material delivery," IEEE Trans. Automation Science and Engineering, vol. 8, issue 4, pp. 683-693, Oct. 2011.

[15] L. E. D. L. Torre, I. S. Dolinskaya, and K. R. Smilowitz, "Disaster relief routing: Integrating research and practice," Socio-Economic Planning Sciences, vol. 46, issue 1, pp. 88-97, March 2012.

[16] G. Leduc, "Road Traffic Data: Collection Methods and Applications," Institute for Prospective Technological Studies, European Commission, Nov. 2008.

[17] http://www.honda.co.jp/news/2011/4110315a.html (in Japanese)

[18] E. W. Dijkstra, "A note on two problems in connexion with graphs," Numerische Mathematik, vol. 1, pp. 269-271, 1959.

[19] D. L. Applegate, R. E. Bixby, V. Chvatal, and W. J. Cook, The Traveling Salesman Problem: A Computational Study, Princeton University Press, 2007. 